\title{
Total electron scattering cross sections from para-benzoquinone in the energy range 1-200 eV
}

\author{
A. I. Lozano, ${ }^{1 *}$ J. C. Oller, ${ }^{2}$ D. B. Jones, ${ }^{3}$ R. F. da Costa,,${ }^{4,5}$ M. T. do N. Varella, ${ }^{6}$ M. H. \\ F. Bettega, ${ }^{7}$ F. Ferreira da Silva, ${ }^{7}$ P. Limão-Vieira, ${ }^{8}$ M. A. P. Lima, ${ }^{4}$ R. D. White, ${ }^{9}$ M. J. \\ Brunger, ${ }^{3}$ F. Blanco, ${ }^{10}$ A. Muñoz ${ }^{2}$, and G. García, ${ }^{1 *}$
}

\footnotetext{
${ }^{1}$ Instituto de Fisica Fundamental, CSIC, Serrano 113-bis, E-28006 Madrid, Spain

${ }^{2}$ Centro de Investigaciones Energéticas Medioambientales y Tecnológicas (CIEMAT), Avenida

Complutense 22, 28040 Madrid, Spain
}

${ }^{3}$ College of Science and Engineering, Flinders University, GPO Box 2100, Adelaide, SA 5001,

Australia

"Instituto de Física "Gleb Wataghin", Universidade Estadual de Campinas, 13083-859,

Campinas, São Paulo, Brazil

${ }^{5}$ Centro de Ciências Exatas, Departamento de Física, Universidade Federal do Espírito Santo, 29075-910, Vitória, Espírito Santo, Brazil

${ }^{6}$ Instituto de Física, Universidade de São Paulo, Rua do Matão 1731, 05508-090 São Paulo, São Paulo, Brazil

${ }^{7}$ Departamento de Física, Universidade Federal do Paraná, CP 19044, 81531-990, Curitiba, Paraná, Brazil

${ }^{8}$ Laboratório de Colisões Atómicas e Moleculares, CEFITEC, Departamento de Física, Universidade NOVA de Lisboa, 2829-516 Caparica, Portugal

${ }^{9}$ College of Science and Engineering, James Cook University, Townsville, Australia

${ }^{10}$ Departamento de Física Atómica, Molecular y Nuclear, Universidad Complutense de Madrid, 28040 Madrid, Spain 


\begin{abstract}
Total electron scattering cross sections, from para-benzoquinone, for impact energies ranging between 1 to $200 \mathrm{eV}$, have been obtained by measuring the attenuation of a linear electron beam under magnetic confinement conditions. Random uncertainty limits on these values have been found to be within 5\%. Systematic errors, due to the axial magnetic beam conditions in combination with the acceptance angle of the detector, have been evaluated by integrating our calculated independent atom model with the screening corrected additivity rule and interference term elastic differential cross sections over that detection acceptance angle. Our previous calculations and measurements on this molecule (Jones et al. J. Chem. Phys. 148, 124312 (2018) and J. Chem. Phys. 148, 204305 (2018)), have been compiled and complemented with new elastic and inelastic scattering cross section calculations in order to obtain a comprehensive cross section data base, within the considered energy range, for modelling purposes. The self-consistency of the present data set has been evaluated by simulating the electron transport of $15 \mathrm{eV}$ electrons in para-benzoquinone, and comparing those results with the observed transmitted intensity distribution.
\end{abstract}

Keywords:

Electron transmission-beam measurements, para-benzoquinone, electron scattering cross sections, transport simulations

*Corresponding authors: Email addresses: anita_ilm@iff.csic.es, g.garcia@csic.es

\title{
1. Introduction
}

Benzoquinones play an important role in biological systems due to their relevance in the reversible reduction mechanism in key cellular processes, ${ }^{1}$ as well as in photosynthesis ${ }^{2,3}$. Quinone derivatives are also being explored as a low-cost, sustainable material that can be used in energy harvesting and storage devices. ${ }^{4,5}$ Para-benzoquinone $\left(\mathrm{pBQ}, \mathrm{C}_{6} \mathrm{H}_{4} \mathrm{O}_{2}\right)$ is the simplest quinone, and therefore serves as the ideal prototypical structure for understanding the electronic properties and mechanisms of quinone chemistry. Electron scattering cross sections from quinones have therefore attracted some attention. Low-energy dissociative electron interactions have been reviewed by Ómarsson and Ingólfsson, ${ }^{6}$ while electronic excitation and ionisation processes have been studied by several different authors. ${ }^{2,3,7-15}$ Electron attachment processes have also been experimentally studied and some resonances identified, ${ }^{16,17,18,19}$ with some of those resonances having recently been investigated through R-matrix ${ }^{20}$ and "ab initio" electronic structure calculations of the $\mathrm{p}-\mathrm{BQ}$ anion. ${ }^{21,22}$ More recently, we have combined theoretical and experimental photoabsorption techniques to investigate the electronic excitedstate structure of $\mathrm{pBQ}$, and their corresponding electron scattering cross sections, together with some elastic differential cross section calculations. ${ }^{23}$ Within this joint research, elastic scattering and vibrational excitation differential cross sections for electron impact have also been investigated. ${ }^{24}$ In spite of all this considerable work, done in order to obtain comprehensive electron interaction cross section data for modelling important applications (see refs. 2, 3 and references therein), we note that direct measurements of $\mathrm{pBQ}$ 's total electron scattering cross sections (TCS) are not available in the literature. Reliable TCS are considered as reference values to evaluate electron transport models. ${ }^{25}$ In addition, being the sum of the contribution of all 
possible processes (open channels) at a given impact energy, they are key parameters to check the consistency of the available integral cross section scattering data.

These latter considerations motivated the present study in which, utilizing a recently reported state of-the-art electron scattering apparatus, ${ }^{26}$ TCS values from $\mathrm{pBQ}$, for electron impact energies ranging from 1 to $200 \mathrm{eV}$, have been determined with overall uncertainties to within $7 \%$. The previous electron scattering data have also been compiled here, and the consistency between the available integral cross sections and the present TCS values is discussed. In addition, we report on new elastic electron scattering cross sections for energies ranging from 1 to $12 \mathrm{eV}$ calculated with the Schwinger multichannel method (SMC) at the 1ch-SEP (1-open channel and 88-closed channels) level of approximation, as well as calculated inelastic electronic-state excitation and total ionisation cross sections. These latter cross sections are calculated with our independent atom model with the screening corrected additivity rule (IAMSCAR) procedure. Interference terms (I) are also included as a part of that procedure. This analysis will provide a complete data set for modelling electron interactions in the considered energy range (1-200 eV), with an example of cross section data validation through Monte Carlo simulation techniques being presented by modelling the electron transport of $15 \mathrm{eV}$ electron interactions in $\mathrm{PBQ}$ and comparing the simulated energy distribution of the transmitted intensity with that experimentally obtained.

The structure of the remainder of this paper is as follows. In section 2 we detail the experimental and theoretical methods used in this study, together with an analysis of the corresponding measurement uncertainties. Our results are then presented, discussed and compared with the available data in section 3, with an example of modelling electron transport in $\mathrm{pBQ}$ being given in section 4. Finally, some conclusions from this study are summarised in section 5 .

\section{Experimental and theoretical methods}

\subsection{Transmission-beam attenuation measurements}

The experimental apparatus and techniques used for the present transmission-beam attenuation study have recently been described ${ }^{26}$ and so will not be detailed again here. Briefly, a linear electron beam is confined by an intense (typically 0.1 Tesla) axial magnetic field which converts any scattering event into a kinetic energy loss in the forward direction, i.e. parallel to the magnetic field (see Ref. 26 for full details). The primary electron beam, generated by an emitting filament, is cooled and confined in a magnetic nitrogen gas trap (GT) which reduces the initial energy spread of $500 \mathrm{meV}$ down to about 100-200 meV. Pulsed voltages applied to the trap electrodes produce a pulsed electron beam with well-defined energy and narrow energy spread to enter the scattering cell. The scattering chamber (SC) is a $40 \mathrm{~mm}$ long gas cell, defined by two $1.5 \mathrm{~mm}$ diameter apertures, through which the pulsed electron beam passes when the $\mathrm{pBQ}$ pressure inside the chamber is varied from 0 to 5 mTorr (as measured by a MKS-Baratron 627B absolute capacitance manometer). Electrons emerging from the SC are analysed in energy by a retarding potential analyser (RPA) and finally detected by a double microchannel plate (MCP) electron multiplier operating in single counting mode. The total cross section $\left(\sigma_{T}\right)$ is determined from the transmitted intensity, which follows the well-known Lambert-Beer attenuation law for ideal gases:

$$
\ln \left(\frac{I}{I_{0}}\right)=-L \sigma_{T} n=-\frac{L \sigma_{T}}{k T} p,
$$


where $I$ is the transmitted electron intensity, $I_{0}$ the initial intensity, $n$ the molecular gas density, $L$ is the interaction region length, $k$ is Boltzmann's constant, $T$ is the absolute temperature and $p$ is the gas pressure. $T$ is derived from $T=\sqrt{T_{c} T_{m}}$, where $T_{c}$ and $T_{m}$ are the temperature of the scattering chamber measured with a thermocouple and the temperature of the Baratron gauge. Measurement conditions, data acquisition and data analysis are controlled by a custom designed LabView (National Instrument) programme.

For each incident electron energy, attenuation measurements were repeated at least 5 times in order to ensure that statistical uncertainties remained below $4 \%$. Other random uncertainties are related to the temperature measurement (within 1\%, according to manufacturer's data) and the numerical fitting procedure (about $1 \%$ ). By combining these uncertainties, a total uncertainty limit of $5 \%$ has been determined for the present measurements. Systematic errors linked to the experimental technique are those connected to the so-called "missing angles". Due to the magnetic field confinement, the energy resolution determines the acceptance angle of the detector. As detailed in Ref. 26, and also in Fuss et al. ${ }^{27}$ and Sanz et al. ${ }^{28}$, the magnitude of this systematic error can be evaluated from the theoretical data by integrating the calculated differential elastic cross sections (DCS) over the "missing" experimental angles. This effect is especially important for polar molecules, but this is not the case here as $\mathrm{pBQ}$ has no permanent dipole moment. The significance of this error source in the present experimental results will be discussed in section 3 .

Perhaps of most importance here is to note that prior to making our $\mathrm{pBQ}$ total cross section measurements, the performance of the new apparatus and our measurement techniques were thoroughly benchmarked against the known TCS values of $\mathrm{N}_{2}{ }^{29,30}$ over the energy range of interest. Excellent agreement between our measured TCS data for $\mathrm{N}_{2}$ and the established values $^{29,30}$ was found, giving us confidence in the validity of the TCS we have subsequently measured for $\mathrm{pBQ}$.

\subsection{IAM-SCAR+I calculation procedure}

Our independent atom model with screening corrected additivity rule method, including interference effects (IAM-SCAR+I), has been utilised to calculate differential and integral elastic as well as integral inelastic (electronic excitation and ionisation) cross sections for impact energies ranging from 1 to $200 \mathrm{eV}$. The calculation procedure and details on its application to pBQ molecules can be found in our previous studies ${ }^{2,3,23,24}$ and references therein, thus we will omit them in this article. Here we simply mention that including interference effects into the IAM-SCAR representation results in a clear magnitude increment of the differential elastic cross section for the smaller scattering angles, ${ }^{31}$ which consequently represents a magnitude increment in the corresponding integral elastic scattering cross sections. We have recently shown that for similar benzene-like based molecules, such as pyridine, this increment can be of the order of $25-30 \%$ for the higher energies. ${ }^{32}$ The IAM-SCAR procedure has provided reasonable agreement for a wide variety of molecular targets for energies above $\sim 20 \mathrm{eV},{ }^{33}$ but the role of the new interference terms still needs some further experimental validation. Below $20 \mathrm{eV}$ the IAM-SCAR+I method is not generally accurate enough to describe electron scattering processes from molecules, but this low-energy limit depends on the target structure and requires a careful investigation for each particular case. Within this model inelastic scattering processes are not affected by the interference terms and they are calculated as a whole from the imaginary part (absorption) of the interaction potential. However, as described in a recent article, ${ }^{34}$ by alternately using as the threshold energy of the absorption potential either the lowest 
electronic-state excitation energy or the ionisation energy we are able to extract the integral excitation and the total ionisation cross sections from the calculated integral inelastic cross sections. We have recently seen that total ionisation cross sections of some organic molecules, ${ }^{35,36}$ as derived from this procedure, are in fairly good agreement with the available experimental results.

\subsection{Schwinger multichannel method (SMC)}

The Schwinger multichannel (SMC) method ${ }^{37}$ for electron-molecule scattering is a variational approach especially designed to deal with targets of arbitrary geometries. The method takes into account important effects such as the electron exchange (SE), the electron-target polarisation interaction (SEP), and it is also able to incorporate flux competition between the elastic and inelastic channels through electronic multichannel coupling ( $N_{\text {opench-SEP), where }}$ Nopen denotes the number of energetically open electronic states. The high computational cost in getting meaningful results for polyatomic targets was accounted for by the use of parallel computing ${ }^{38}$ in an implementation that also employs norm-conserving pseudopotentials (SMCPP) ${ }^{39}$ and single-excitation configuration interaction techniques for the target description. ${ }^{24}$ The method has recently been reviewed, ${ }^{40}$ in a paper which also describes in detail our implementation of the SE and SEP approaches, and applied to $\mathrm{pBQ}$ molecules for impact energies above $15 \mathrm{eV}$ using an up to 89-channel close-coupling scheme. ${ }^{2,3,23}$ Though, not all the details of this calculation procedure need to be given here, it is important to call attention to the fact that it was developed so as to provide a good description of electron scattering by $\mathrm{pBQ}$ for energies above $15 \mathrm{eV}^{2,3}$ where all states that become energetically allowed to the target are in fact treated as open channels. At this level of approximation the target is only slightly polarized due to the presence of the states that remains as closed channels in the calculation. In order to describe the interaction of electrons with $\mathrm{pBQ}$ in the energy region below 5-7 eV a more sophisticated treatment for the polarisation effects should certainly be given so as to provide an adequate description for the formation of resonant states. Note that those resonance states represent the temporary capture of the incident electron by the molecular potential. Due to the limitation of our IAM$\mathrm{SCAR}+\mathrm{I}$ procedure at lower energies $(<20 \mathrm{eV})$, we have carried out a new SMCPP calculation on $\mathrm{pBQ}$ for the lower impact energies, ranging from 1 to $12 \mathrm{eV}$. As elastic scattering is the dominant channel for such fairly low energies, and the main purpose of this study is to provide as quick as possible, but still accurate, electron scattering calculation results that are ready to be used in modelling procedures, this new SMCPP calculation has been performed at the 1ch-SEP (1-open channel and 88-closed channels) level of approximation. Note that we have checked its reliability by comparing the simulation predictions with the observed energy distribution of transmitted electron intensities. This facet of our investigation is described later in section 4.

\section{Results and discussion}

The total electron scattering cross sections $\left(\AA^{2}\right)$, as measured with the experimental set-up described in section 2.1, are shown in Table 1 together with the present IAM-SCAR+I integral scattering cross sections (elastic, excitation, ionisation and total) and SMCPP (1-ch-SEP) elastic ICS.

Table 1. Integral and total electron scattering cross sections from para-benzoquinone (in $\AA^{2}$ units). The errors cited are the random uncertainties only. 


\begin{tabular}{|c|c|c|c|c|c|c|c|}
\hline \multirow[t]{3}{*}{$E(e V)$} & \multicolumn{2}{|c|}{ Experiment } & \multicolumn{5}{|c|}{ Calculations } \\
\hline & \multirow[t]{2}{*}{ TCS } & \multirow{2}{*}{$\begin{array}{l}\text { Uncertainty } \\
\text { (\%) }\end{array}$} & \multicolumn{4}{|c|}{ IAM-SCAR+I } & \multirow{2}{*}{$\begin{array}{l}\text { SMCPP } \\
\text { (1-ch-SEP) } \\
\text { Elastic }\end{array}$} \\
\hline & & & TCS & Ionisation & Excitation & Elastic & \\
\hline 1 & 38.7 & 4.2 & 106 & & & 106 & 32.1 \\
\hline 1.3 & 40.5 & 1.2 & & & & & 47.1 \\
\hline 1.5 & 40.1 & 3.5 & 88.8 & & & 88.8 & 37.4 \\
\hline 1.8 & 47.9 & 2.8 & & & & & 48.0 \\
\hline 2 & 44.8 & 3.0 & 79.8 & & & 79.8 & 65.4 \\
\hline 2.5 & 38.2 & 1.5 & & & & & 51.8 \\
\hline 3 & 39.3 & 3.6 & 71.4 & & & 71.4 & 43.7 \\
\hline 3.6 & 39.7 & 2.9 & & & & & 39.5 \\
\hline 4 & 48.1 & 3.1 & 66.9 & & & 66.9 & 40.6 \\
\hline 4.5 & 54.3 & 1.0 & & & & & 40.0 \\
\hline 5 & 54.1 & 2.0 & 64.1 & & & 64.1 & 39.9 \\
\hline 5.5 & 63.5 & 2.3 & & & & & 40.4 \\
\hline 6 & 59.7 & 1.5 & & & & & 40.9 \\
\hline 7 & 52.4 & 2.2 & 61.3 & & & 61.3 & 45.0 \\
\hline 8 & 54.3 & 4.0 & & & & & 43.9 \\
\hline 9 & 53.6 & 3.5 & & & & & 49.4 \\
\hline 10 & 58.3 & 3.0 & 59.4 & & 0.281 & 59.1 & 48.4 \\
\hline 12 & 59.0 & 4.2 & & & & & 46.3 \\
\hline 15 & 65.1 & 2.6 & 58.2 & 0.479 & 3.16 & 54.6 & 48.9 \\
\hline 20 & 61.3 & 1.3 & 57.7 & 3.44 & 5.60 & 48.7 & 42.5 \\
\hline 30 & 59.6 & 3.9 & 57.1 & 9.67 & 5.43 & 42.0 & 38.3 \\
\hline 40 & 58.5 & 3.0 & 54.9 & 12.35 & 4.75 & 37.8 & 32.7 \\
\hline 50 & 55.9 & 2.9 & 52.8 & 13.6 & 4.50 & 34.7 & 28.5 \\
\hline 70 & 48.1 & 4.9 & 47.6 & 13.7 & 3.72 & 30.2 & \\
\hline 100 & 42.0 & 5.0 & 42.6 & 13.1 & 3.30 & 26.2 & \\
\hline 150 & 34.4 & 4.8 & 36.7 & 11.8 & 3.02 & 21.9 & \\
\hline 200 & 30.0 & 3.5 & 32.5 & 10.6 & 2.77 & 19.1 & \\
\hline
\end{tabular}

The present uncertainty limits have been derived by a root mean square quadratic combination of all the random uncertainty sources described in section 2.1 (see Ref. 26 for a comprehensive analysis of these uncertainty sources). From a careful inspection of Table 1 , these limits range from 1 to 5\%, depending on the incident energy. Possible systematic errors are described later with a fuller discussion for them being found in Refs. 26-28. We have also checked that the actual absorption length $(L)$ corresponds to the geometrical length of the scattering chamber, by measuring the well-known electron scattering TCS for molecular nitrogen at selected energies, finding (as noted earlier) excellent agreement with the benchmark values available in the literature (see Ref. 25 for details). The electron intensity count rate was less than $10^{3} \mathrm{~s}^{-1}$ (i.e. less than $10^{-16} \mathrm{~A}$ electron current). Under these conditions no dependence of the measured TCS on the electron current was found, so ensuring that possible space charge effects are negligible in this experiment. In order to ensure that multiple scattering processes are absent under our working conditions, attenuation measurements were performed at very low gas pressure (from 0 to less than $2 \mathrm{mTorr}$, depending on the incident energy). To illustrate this dependence, a typical attenuation curve for $15 \mathrm{eV}$ incident electron energy is shown in Fig.1. 


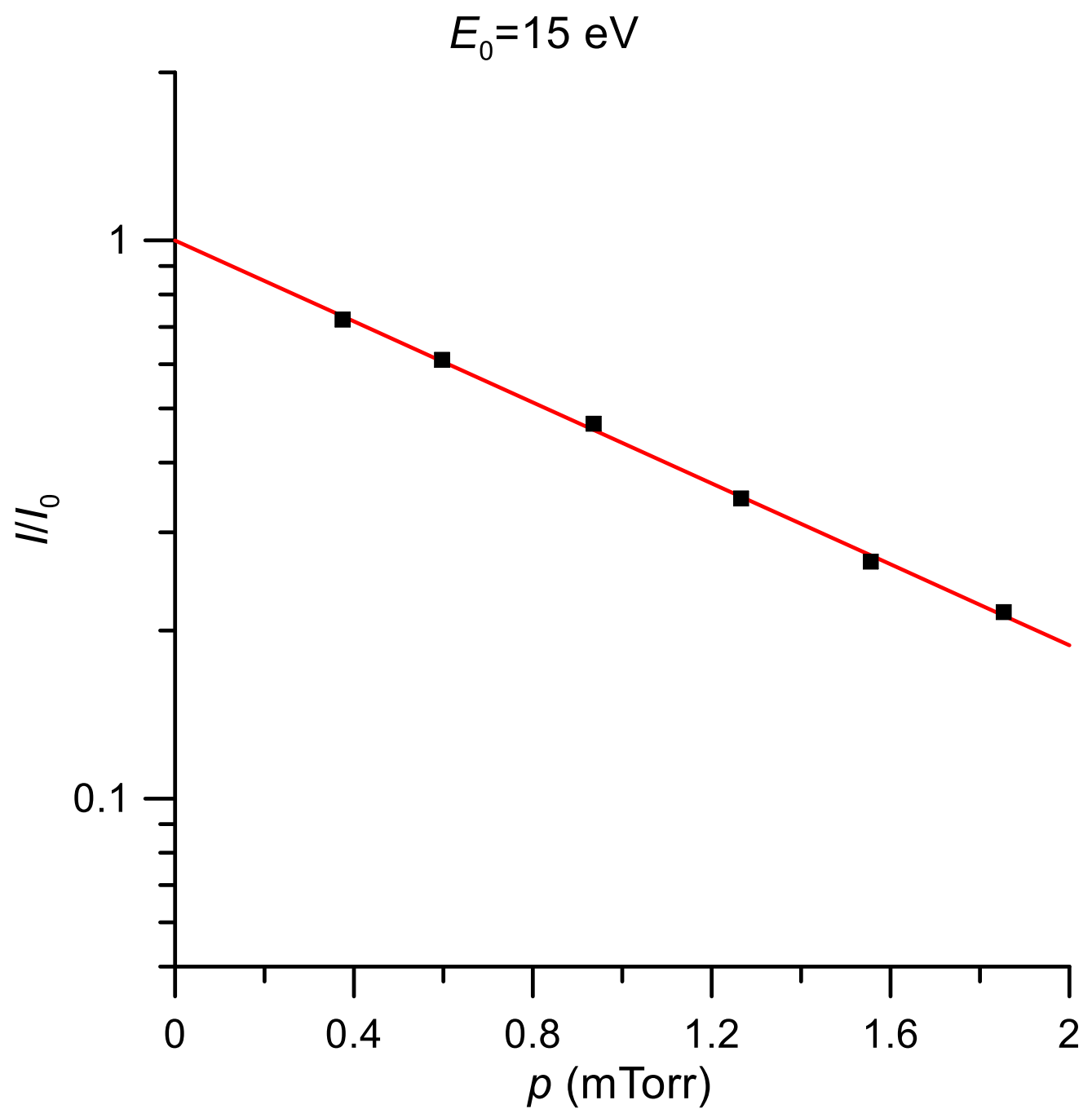

Fig. 1. Typical attenuation curve, for $15 \mathrm{eV}$ incident electron energy, as a function of the $\mathrm{pBQ}$ pressure in the SC. Note the logarithmic $y$-axis.

The maximum gas pressure used for this energy is 1.7 mTorr and the attenuation curve nicely fits to a single logarithmic function, proving that multiple scattering effects are not affecting the present results. The incident energy was calibrated against the well-known resonance energy corresponding to the first peak in the $N_{2}$ TCS, which is largely due to the $v=0-1$ vibrational excitation of $\mathrm{N}_{2}{ }^{41}$ As shown in Ref. 26, the energy resolution of the incident electron beam $(\Delta E)$, derived from the transmitted electron profiles on the RPA, is typically within 100-200 meV. However, biasing the RPA to the higher energies, to reduce the transmitted intensity by $25 \%$ of that for the incident electron intensity, an effective energy resolution better than $100 \mathrm{meV}$ was customarily achieved. ${ }^{26}$ Additionally, and as explained in Refs. 26-28, due to the axial magnetic field conditions of the present experiment, the energy resolution and the incident energy $(E)$ are linked to the angular resolution $\left(\Delta \theta^{\circ}\right)$ as follows:

$\left(\Delta \theta^{\circ}\right)=\arccos \sqrt{1-\Delta E / E}$.

Note that electrons elastically scattered within $\Delta \theta^{\circ}$ and $180^{\circ}-\Delta \theta^{\circ}$ are, for the MCP detector, indistinguishable from the unscattered electrons, and constitute the main systematic error source of the present measurements. This effect always tends to lower the measured cross 
sections from their "true" value, and their magnitude can be estimated by integrating the calculated DCS from $0^{\circ}$ to $\Delta \theta^{\circ}$ and from $180^{\circ}-\Delta \theta^{\circ}$ to $180^{\circ}$. Using both our IAM-SCAR+I and SMCPP calculated elastic DCSs and integrating these values over the angular ranges just noted, we obtain that the contribution of this effect to the total cross section is less than $2-4 \%$, depending on the incident energy. These magnitudes are within the quoted random uncertainty limits, but if we combine these with those random uncertainty limits we obtain overall uncertainty limits for the present measurements from 4 to $7 \%$, for incident energies from 200 down to $1 \mathrm{eV}$, respectively.

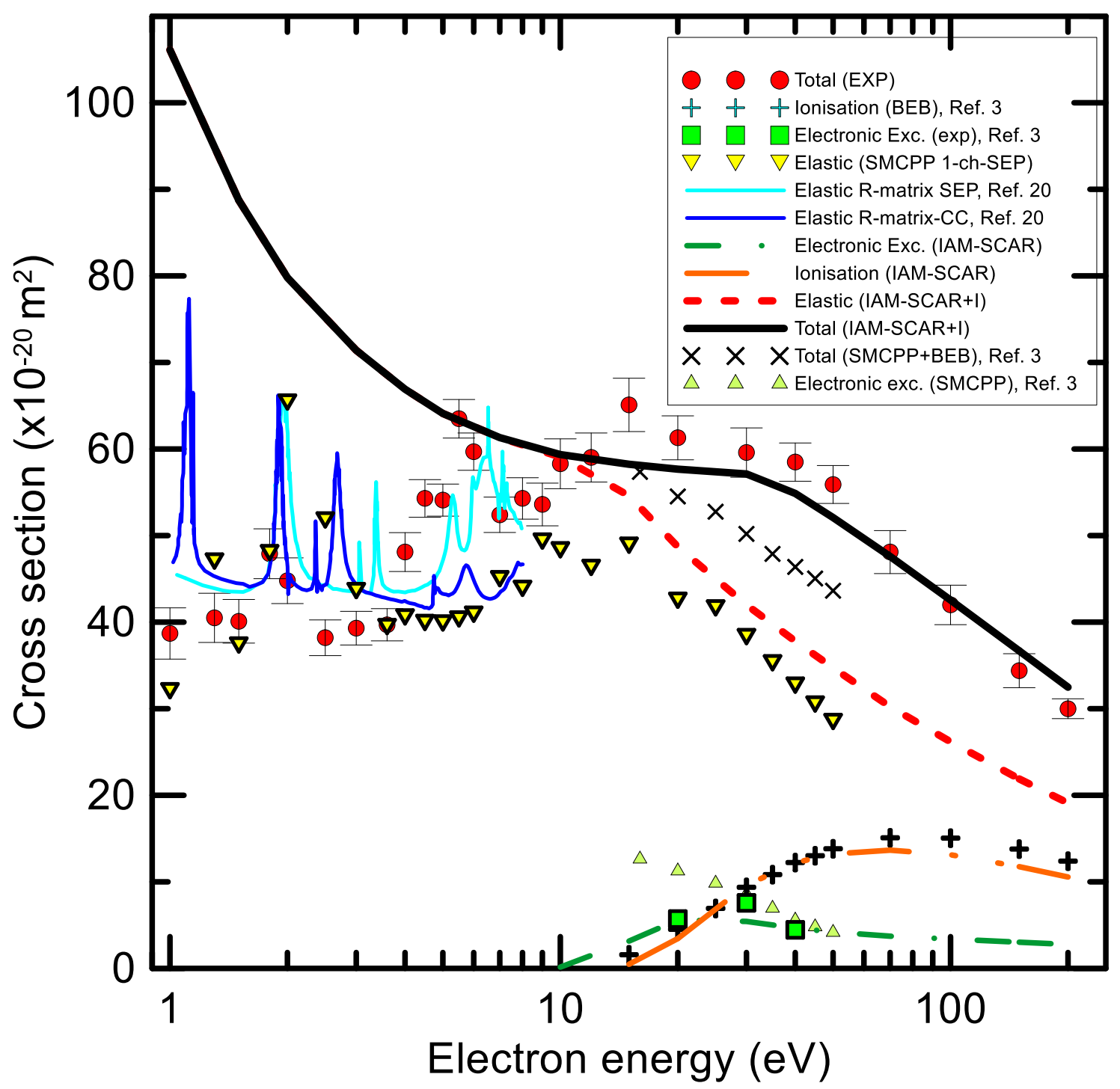

Fig.2. Integral and total electron scattering cross sections from $\mathrm{pBQ}$. See legend in the figure.

In order to facilitate our discussion, the present theoretical and experimental integral (elastic, excitation, ionisation) and total cross sections are plotted in Fig. 2 together with our recently published experimental total excitation cross sections ${ }^{3}$ and calculated BEB total ionisation and SMPP+BEB total cross sections. ${ }^{3}$ From 10 to $200 \mathrm{eV}$ the agreement between the present experimental TCS and the corresponding IAM-SCAR+I calculation is very good, except for the observed increase of the measured TCS at around $12 \mathrm{eV}$ which is not followed by the calculation. Note that this energy is near to the $\mathrm{pBQ}$ threshold ionisation energy $(10.0 \pm 0.1 \mathrm{eV})^{13}$ and our calculation, which is based on the atomic ionisation energies, is not able to properly reproduce near-threshold molecular scattering processes. However, from $12 \mathrm{eV}$ and above our calculated 
total ionisation and total excitation cross sections agree well with our previous BEB calculation and experimental total electronic-state excitation cross sections. ${ }^{3}$ From 15 to $50 \mathrm{eV}$ additional data for the elastic, electronic excitation ICS and TCS are given in Ref. [3] and are derived from our SMCPP calculations, also in combination with the BEB ionisation cross sections for the TCS. These data are also plotted in figure 2 , but they show some inconsistences with the IAM-SCAR+I calculation which is considered a good reference for these relatively high energies (see Ref. [3] for further details). As expected, for energies below $10 \mathrm{eV}$, our IAM-SCAR+I representation overestimates the magnitude of the experimental data. In the 1-10 eV incident energy range the molecular orbital structure, not considered in the IAM-SCAR+I representation, is essential and thus more sophisticated techniques are required in order to reproduce the observed scattering cross section values and any resonances that may exist. In order to analyse how the pBQ molecular structure may dictate the behaviour of the elastic integral cross section, we therefore performed additional calculations using the SMC method. As shown in Fig. 2 for energies above $15 \mathrm{eV}$, there is a reasonable agreement, within $20 \%$, between our elastic ICSs calculated with both methods. We can therefore conclude that from 15 to $200 \mathrm{eV}$ the present elastic, inelastic and total electron scattering cross sections are self-consistent and ready to be used for modelling purposes.

In the energy range 1-10 eV we can distinguish two different sub-regions. As shown in Fig. 1 of Ref. 2, between 4 and $10 \mathrm{eV}$ the different electronic-state excitation channels are subsequently being opened producing the corresponding increase of the cross section magnitude at the corresponding threshold energies. Our experimental results show increments on the total cross sections just above some particular energies: $4,5,7 \mathrm{eV}$, with the corresponding local maxima at $4.5 \pm 0.2,5.5 \pm 0.2,8.0 \pm 0.2 \mathrm{eV}$, which are coincident with the opening channel sequence: 4,5 , 7 and $7.5 \mathrm{eV}^{2}{ }^{2}$ The R-matrix SEP-results ${ }^{20}$ show some features in this energy range which according to Loupas and Gorfinkiel ${ }^{20}$ might correspond to core-excited resonances. However, the SEP level of approach is not considered reliable enough to accurately define the position of these resonances. Their CC R-matrix calculation, ${ }^{20}$ which includes excited states, is considered in Ref. [20] as more accurate for this purpose. As shown in Fig. 2, this calculation presents three broad features centred around 4.8-5.0, 5.5-6.0 and 7.5-8 eV. These features have been considered as a mixture of core-excited shape and mixed shape core-excited resonances (see Ref. 18 for details). Most of these resonances were previously predicted by Cheng and Huang ${ }^{42}$ using the stabilization method ${ }^{42}$ and the position of some of them have been experimentally confirmed at 4.2-4.4 $4^{16,17,43}$ and $5.8 \mathrm{eV},{ }^{17}$ respectively. These positions agree reasonably well with two of the present TCS local maxima and our third feature at $8.0 \pm 0.1 \mathrm{eV}$ can be correlated with the 7.82-7.98 eV energy of the $2^{2} B_{2 g}$ excited state of the $\mathrm{p}-\mathrm{BQ}$ vertical anion calculated in Ref. 42. The positions of these resonances are also consistent with the experimental electronic-state excitation thresholds of $4.07^{43}, 5.12^{44}$ and $7.1^{45} \mathrm{eV}$ corresponding to the $1^{1} B_{3 \mathrm{~g}}, 1^{1} B_{1 \mathrm{u}}$ and $2^{1} B_{1 \mathrm{u}}$ states of $\mathrm{pBQ}$, respectively. ${ }^{20}$

Below $4 \mathrm{eV}$, according to the energy loss functions shown in Ref. [23], electronic-state excitation cross sections are expected to be very small in magnitude and so less relevant than for those in the range $4-15 \mathrm{eV}$ in the simulation. Possible structures in the total cross sections may be attributed to resonance decay into any or all of the electron attachment (EA), vibrational excitation or elastic channels. As already mentioned, Loupas and Gorfinkiel ${ }^{20}$ have recently published a systematic study to identify electron scattering resonances for $\mathrm{pBQ}$ in the lowenergy domain. Using an application of the $R$-matrix method for molecules ${ }^{46}$ they identified several shape, Feshbach and core-excited resonances in the 0-8 eV energy range. In particular, for incident energies below $4 \mathrm{eV}$, the positions and widths of the low-lying resonances were 
calculated at the static-exchange-polarisation (SEP) and close-coupling (CC) levels. ${ }^{20}$ According to Fig. 2, we experimentally observed two local maxima of the TCS in this region, a very weak structure at $1.3 \pm 0.2$ and a stronger structure at $1.8 \pm 0.2 \mathrm{eV}$. These energies are in excellent agreement with the $1.35 \mathrm{eV}$ and $1.90 \mathrm{eV}$ features reported by Cooper et al. ${ }^{16}$ which correspond to the energy of the maximum $\mathrm{C}_{6} \mathrm{H}_{4} \mathrm{O}_{2}{ }^{-}$and $\mathrm{C}_{5} \mathrm{H}_{4} \mathrm{O}_{2}^{-}$negative ion production cross section for $\mathrm{p}$ BQ. ${ }^{16}$ This good agreement seems to indicate that the above two resonances that we found in the present TCS values are due to electron attachment processes. Additionally in Fig. 2 , although our 1ch-SEP calculation does not in principle allow an appropriate analysis to assign the character of the structures that appear in its elastic ICS, it seems to reproduce well the position of the first resonance $(1.3 \mathrm{eV})$ and predicts a maximum cross section value at $2 \mathrm{eV}$ for the second resonance, which is in reasonable agreement with the experimental result.

Previous calculations indeed present a broad spread of resonance positions in their respective results (see Ref. 20 and references therein). For our $1.3 \mathrm{eV}$ resonance, the most recent R-matrix results of Loupas and Gorfinkiel ${ }^{20}$ report a feature at $0.85 \mathrm{eV}$ and $1.1 \mathrm{eV}$, when using the SEP and $\mathrm{CC}$ levels, respectively. Our second resonance at $1.8 \pm 0.2 \mathrm{eV}$ is calculated at energy values being 3.36 and $2.67 \mathrm{eV}$ at the SEP and CC levels, respectively. Those energies are respectively about $46 \%$ and $33 \%$ higher than our experimental results. The other lower-energy $(<4 \mathrm{eV})$ resonances proposed in the calculations of Ref. 20 are not observed in the present experiment, although in some cases this may be the result of our finite energy resolution "washing" them out. Resonances analysed in Ref. 20 are also assigned to different symmetries (see Ref. 20) for further information). Recent accurate "ab initio" calculations from Kunitsa and Bravaya ${ }^{21}$ together with their revisited $\mathrm{pBQ}$ radical anion electronic structure calculation ${ }^{22}$ located a resonance at 2.55 $\mathrm{eV}$ which they assigned to $\mathrm{a}^{2} \mathrm{~A}_{u}$ shape resonance and proposed as being the gateway for electron attachment in redox processes involving quinones. ${ }^{21}$ This result is supported by the observed resonant peak in photodetachment experiments ${ }^{18}$ at $2.5 \mathrm{eV}$. However, that resonance is not visible in our SMCPP-SEP calculation nor in our experimental TCS although we should note that as its calculated and observed widths are 0.013 and $0.025 \mathrm{eV}$, respectively, then as the energy step size of the present SMCPP-SEP 1 channel calculation and the energy resolution of the present measurements are not very fine it is not surprising that we do not to distinguish such narrow structures.

\section{Electron transport simulation}

One of the goals of this experimental and theoretical study is to provide benchmarked electron scattering cross section data, to be used in modelling electron transport. ${ }^{47}$ In particular electrontransfer reactions involving $\mathrm{pBQ}$ are relevant for biomedical applications requiring precise anion formation models ${ }^{48}$ as well as for plasma processing simulation of possible precursors. ${ }^{49}$ For example, some quinones ${ }^{18}$ are key acceptors in the electron transport chains of photosynthesis ${ }^{50}$ and respiration. ${ }^{51}$ From the discussion of the previous section we can conclude that for energies above $15 \mathrm{eV}$ the present combined experimental and theoretical cross sections constitute a selfconsistent data set for the integral elastic, electronic-state excitation and ionisation channels and the total scattering cross section, and are thus ready to be used in modelling simulations. For lower energies, our IAM-SCAR+I calculation does not apply properly and although the present TCS measurement is consistent with our new SMC elastic scattering results, some discrepancies found in the position and magnitude of the resonances predicted by the R-matrix calculation $^{20}$ and the fact of missing the additional resonance as calculated in Ref. 21 , suggest 
that further complementary techniques should be used to investigate this lower energy region. Note that such low energies are crucial for the aforementioned applications, as the most representative chemical reactions occurring in transport processes are induced by low-energy electrons, in particular those that participate in EA processes. ${ }^{52}$

An efficient procedure to a posteriori validate the assembled differential and integral electron scattering cross sections, in a relevant energy range, is to simulate particle tacks through the medium of interest by means of Monte Carlo ${ }^{53}$ methods. Here we use, as input parameters, the cross section data set we want to validate and then compare the simulated output results with some corresponding measurements. With this purpose in mind we have simulated the transport of a $15 \mathrm{eV}$ electron beam through the scattering chamber, under the same magnetic confinement condition as that used for the TCS measurements, but at a higher gas pressure ( 5 mTorr in this case) to ensure that multiple scattering processes are now taking place. In these conditions, incident electrons are losing energy via elastic and inelastic scattering processes along the $\mathrm{SC}$ and the energy distribution of the emerging electrons will cover the entire energy range from 0 to $15 \mathrm{eV}$. This is therefore a nice way to evaluate our lower energy ( $\leq 15 \mathrm{eV}$ ) electron cross section data base of $\mathrm{pBQ}$. As mentioned in section 2.1, the intense axial magnetic field converts any scattering event into a kinetic energy loss in the axial (parallel to the magnetic field) direction (see Ref.26 for details) and therefore the output of this simulation will also be very sensitive to the assumed differential cross section data set and to the appropriateness of the applied magnetic field intensity. Additionally, if multiple scattering processes occur the validating test becomes more demanding with respect to the accuracy of the cross sections used in order to properly reproduce the observed energy distribution of the transmitted electrons. In the remainder of this section we give some details on our simulation procedure, and a comparison between the simulated and measured outputs.

\subsection{Simulation procedure}

The Low Energy Particle Track Simulation code was developed by our group to model single particle tracks in biologically relevant media. ${ }^{54-57}$ Basically, it is an event-by-event Monte Carlo code which simulates the tracks of charged particles in gaseous and condensed media. This is achieved by sampling the type of interactions, the energy loss and the angular distribution of the scattered particles according to the probability distribution functions derived from the integral cross sections, the energy loss spectra and the differential cross sections, respectively (see Ref. 56 for details). The input data base is formed of a self-consistent set of integral cross sections (elastic and all the inelastic channels that are open in the considered energy range), elastic differential cross sections (i.e. the angular distributions of the elastically scattered electrons), an averaged energy loss spectrum that is representative for the considered energy range (i.e. the energy loss distribution function) and the angular distribution function for inelastically scattered electrons which for each incident energy depends on the corresponding elastic angular distribution and the energy loss according to the empirical procedure described in Ref. 55.

\subsection{Input data for the present simulation}

In order to check the reliability of the present SMC 1ch-SEP calculation, the elastic cross sections derived from this method, excluding the two resonance peaks at 1.3 and $2.0 \mathrm{eV}$ which can be assigned to EA processes, were included into the input data set to be used in the simulation (see Table 2). Note that it is well known that many molecular systems support strong EA cross sections at energies above $\sim 0 \mathrm{eV},{ }^{58-60}$ so that this assignment is by no means fanciful. Considering 
the measured TCS as the reference values representing the integral cross section sum of all the open channels, the difference, at a given energy, between the experimental TCS and the elastic SMC ICS was assumed to be the integral inelastic cross section (IICS). By subtracting from this IICS the experimental vibrational excitation cross section, derived by extrapolating the measured cross sections in Ref. 3 to lower energies based on the energy dependence given in Brunger et al. ${ }^{57}$, and the experimental electronic-state ICSs from Ref. 3, again extrapolated down to their excitation threshold and assuming an excitation function proportional to the interpolated energy loss function, we assigned the resulting values to the remaining EA channel. This process enabled us to derive the attachment cross sections for $E \leq 3 \mathrm{eV}$. For higher energies ( $3 \mathrm{eV} \leq E \leq$ $15 \mathrm{eV}$ ), however, we made use of the relative data in Khvostenko et al. ${ }^{19}$ in order to provide estimates of their attachment cross sections for the simulation. The complete data set obtained with this procedure is shown in Table 2.

Table 2. Set of cross section data (in $\AA^{2}$ units) used for the present Monte Carlo simulation.

\begin{tabular}{|l|l|l|l|l|l|l|}
\hline $\mathrm{E}(\mathrm{eV})$ & TCS (Exp) & $\begin{array}{l}\text { Elastic } \\
\text { (SMCPP } \\
\text { 1ch-SEP) }\end{array}$ & $\begin{array}{l}\text { Electron } \\
\text { attachment }\end{array}$ & $\begin{array}{l}\text { Electronic } \\
\text { excitation }\end{array}$ & $\begin{array}{l}\text { Vibrational } \\
\text { excitation }\end{array}$ & Ionisation \\
\hline 1 & 38.7 & 32.1 & 6.5 & - & 0.12 & - \\
\hline 1.5 & 40.1 & 33.3 & 6.6 & - & 0.22 & - \\
\hline 2 & 44.8 & 34.5 & 9.17 & - & 1.10 & - \\
\hline 3 & 39.3 & 36.8 & 0.24 & - & 2.24 & - \\
\hline 4 & 48.1 & 40.6 & 0.06 & 0.06 & 7.38 & - \\
\hline 5 & 54.1 & 39.9 & 0.33 & 0.119 & 13.8 & - \\
\hline 7 & 52.4 & 45.0 & 0.44 & 0.760 & 6.17 & - \\
\hline 10 & 58.3 & 48.3 & 0.09 & 4.60 & 5.27 & 0.042 \\
\hline 15 & 65.1 & 48.9 & - & 5.00 & 3.30 & 7.91 \\
\hline
\end{tabular}

The main weight of the present simulation is supported by the integral elastic (SMC) cross sections and the upper limit given by the experimental TCS ensures the self-consistency of the remaining inelastic channels which, in this low-energy domain, are less important than the elastic channel. Based on the information in this table, the simulation programme uses validated interpolation numerical procedures ${ }^{55}$ to obtain cross section values between the specific energy values cited in Table 2.

Another challenging point of the current simulation is the presence of the intense $(0.1 \mathrm{~T})$ axial magnetic field along the SC. Note that in these conditions, scattering events with scattering angles close to $90^{\circ}$ lose almost all their kinetic energy within the velocity component parallel to the direction of the beam, which is the energy evaluated by the RPA, and for those scattering angles higher than $90^{\circ}$ the electrons turn back to the cathode where they are reflected and sent back again to the SC. In addition the relatively high pressure used in our current simulation ( 5 mTorr) ensures that multiple scattering processes may occur, making it more difficult for the simulation to reproduce the energy distribution of the electrons finally detected. This clearly indicates the critical dependence of the simulated transmitted spectra on the reliability of the differential cross sections used to derive the angular distribution functions. As an example, Fig. 3 shows the elastic differential cross sections from 1 to $15 \mathrm{eV}$ that we used to derive the elastic angular distribution functions from by a simple normalisation. 


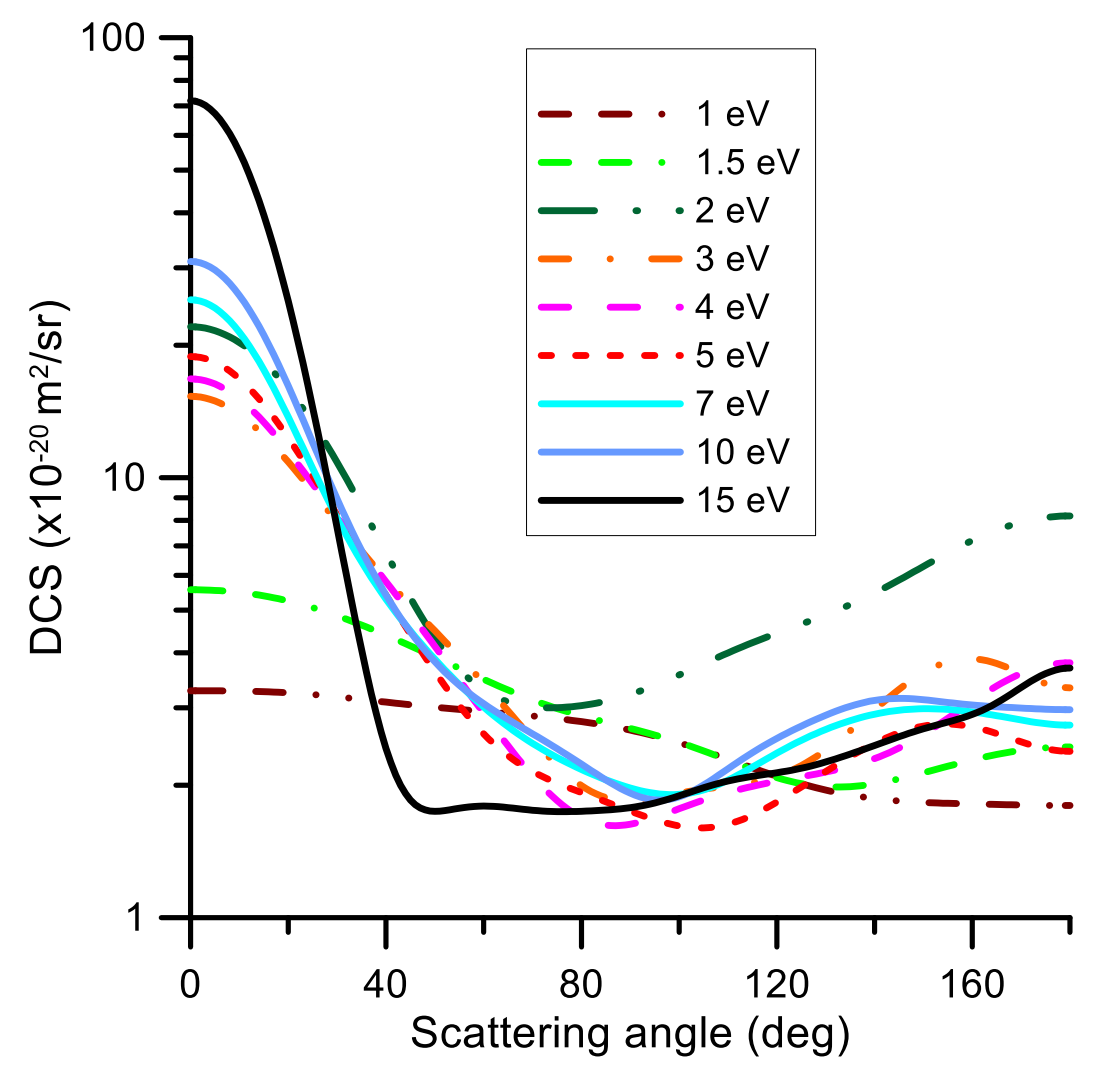

Fig. 3. Elastic differential cross sections $\left(10^{-20} \mathrm{~m}^{2} / \mathrm{sr}\right)$ calculated with the SMCPP method the 1ch-SEP (1-open channel and 88-closed channels) level of approximation, and used to derive the relevant angular distribution functions for the simulation. See also legend in figure.

As mentioned above, the energy loss distribution functions for $\mathrm{pBQ}$ have been generated by averaging experimental energy loss spectra measured in Ref. 3. Fig. 4 represents a plot of the energy loss distribution function employed for $15 \mathrm{eV}$ incident energy, showing the partial distributions for three of the different inelastic channels considered in this simulation: vibrational excitation, electronic-state excitation and ionisation. For DEA processes, incident electrons disappear from the simulation transferring all their kinetic energy to the medium. On the other hand for elastic processes, only kinetic energy is transferred from the incident electron to the target molecule, and its magnitude is determined by their relative mass ratio. Below $1 \mathrm{eV}$ the simulation data are extrapolated down to $0.1 \mathrm{eV}$, after which the electrons disappear from the simulation. 


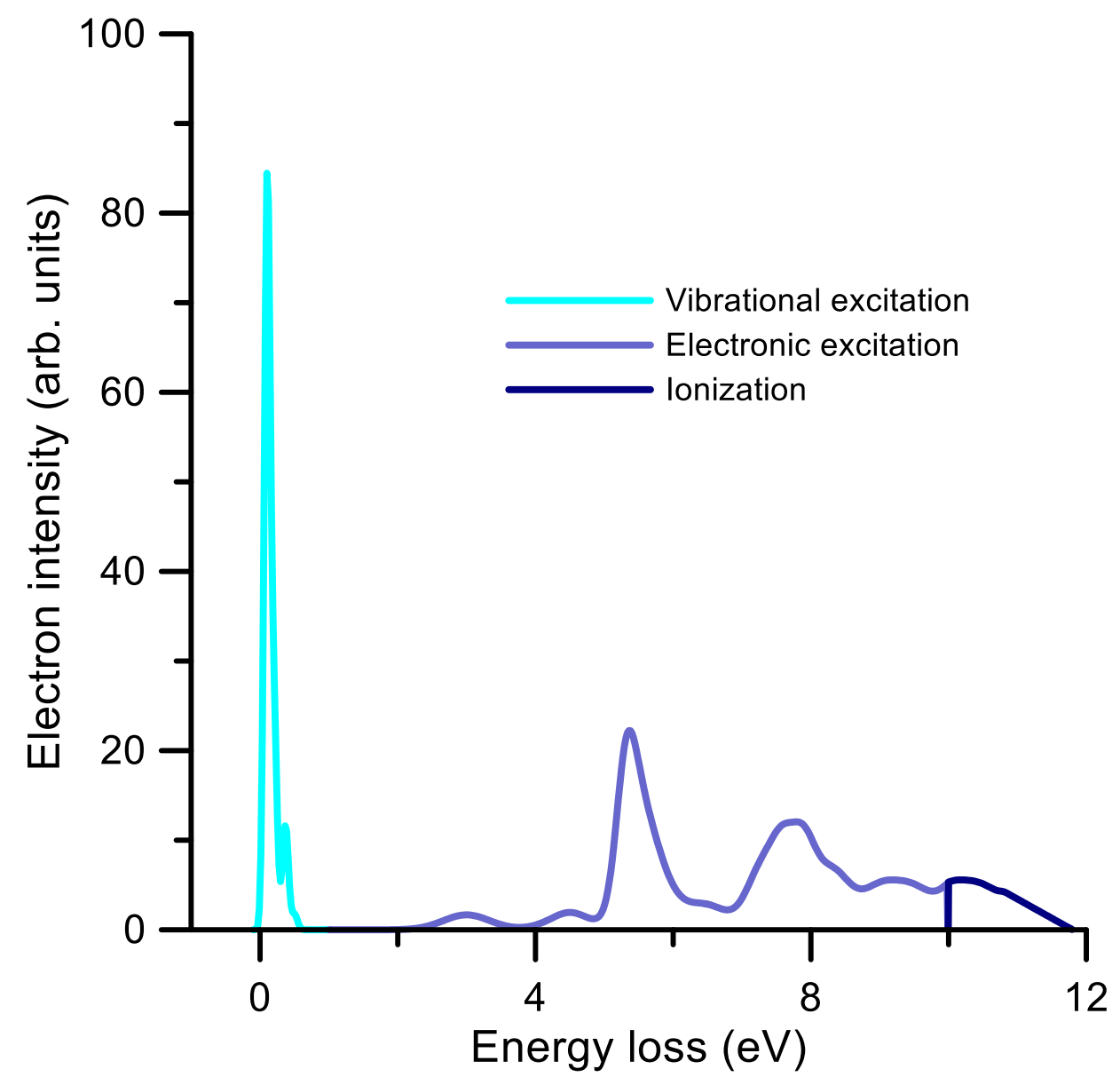

Fig. 4. Energy loss distribution function at $15 \mathrm{eV}$ impact energy, as employed in the present simulation. See also legend in figure.

\subsection{Results of the simulation and comparison with our corresponding measurements}

The simulated energy distributions for electrons transmitted through the $\mathrm{SC}$, under magnetic field confinement conditions, at $\mathrm{pBQ}$ pressures of 0 and $5 \mathrm{mTorr}$ are shown in Fig. 5 . In order to achieve the statistical reproducibility of these results to within $1 \%, 10^{6}$ electrons were run in each simulation. The experimental energy distributions obtained under the same conditions are also plotted in this figure for comparison. As these spectra display the intensity of the electrons passing through the RPA, they represent the integrated energy distribution of the transmitted electrons. 


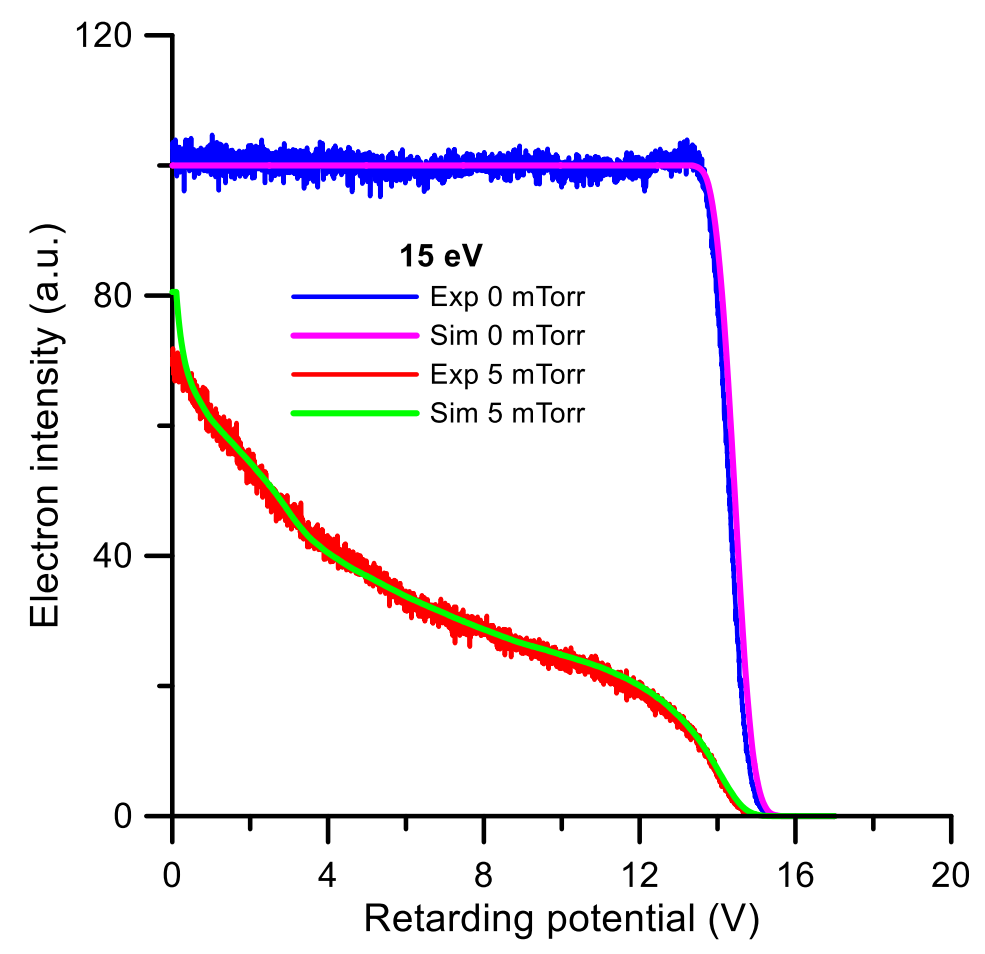

Fig. 5. Simulated versus measured energy distribution for electrons emerging from the SC, as a function of the retarding potential (V). The incident electron energy was $15 \mathrm{eV}$. See also legend in figure.

The 0 mTorr experimental spectrum in Fig. 5 represents the energy distribution of the incident electron beam (without $\mathrm{pBQ}$ gas in the $\mathrm{SC}$ ), and it was analytically fitted in order to obtain the initial electron source distribution for the higher pressure simulation (Sim 5 mTorr in Fig.5). There is an excellent agreement between the simulated and measured energy distributions with $5 \mathrm{mTorr}$ of $\mathrm{pBQ}$ in the $\mathrm{SC}$ over the whole energy range $(0-15 \mathrm{eV})$. Only for energies below about $0.5 \mathrm{eV}$ does the simulated intensity tend to be slightly higher in magnitude than that for the measurements. This is attributed to the difficulties of such low-energy electrons in reaching the detector through the RPA chamber. The good level of agreement we observe in Fig. 5 validates the input data used for the simulation, especially the elastic cross sections, and in particular the DCS values to which the results of this type of simulation are very sensitive. In addition, our present result confirms that the current experimental conditions are appropriate to assume the required axial magnetic confinement ${ }^{26}$ under which any scattering event results in an energy loss in the axial direction, parallel to the magnetic field, and the cyclotron radius ${ }^{26}$ induced by the perpendicular component of the velocity is negligible in comparison with the diameter of the SC limiting apertures.

To provide the reader with another perspective for the significance of this agreement, Table 3 shows the number of interactions computed by the modelling programme in order to obtain the final output and the energy loss in the axial direction, through these interactions, for the $10^{6}$ incident electrons.

Table 3: Summary giving the number of interactions and the total energy loss (eV), as computed with our modelling programme, for the various scattering processes. Note that there were $10^{7}$ incident electrons of $15 \mathrm{eV}$ energy in this simulation.

\begin{tabular}{|l|l|l|l|l|}
\hline Type of process & Number of interactions & $\mathbf{( \% )}$ & Energy loss (eV) & (\%) \\
\hline Elastic & 45983200 & 84.1 & 44784400 & 46.7 \\
\hline
\end{tabular}




\begin{tabular}{|l|l|l|l|l|}
\hline Electron attachment & 998547 & 1.83 & 2168280 & 2.26 \\
\hline Vibrational excitation & 3280080 & 6.00 & 4677950 & 4.87 \\
\hline Electronic excitation & 2125260 & 3.89 & 17330500 & 18.0 \\
\hline Ionisation & 2276160 & 4.16 & 27109700 & 28.2 \\
\hline
\end{tabular}

The results in Table 3 show the importance of elastic scattering in the type of simulation we have carried out here. Using these values, we have plotted in Fig. 6 (a) the number of interactions and (b) the energy loss along the axis per incident particle. It is clear from Fig. 6 (a) that for $15 \mathrm{eV}$ incident electrons and $5 \mathrm{mT}$ Torr of $\mathrm{pBQ}$, multiple scattering processes only occur in the SC for elastic scattering. In addition, on average, we find that each incident electron suffers $\sim 4.5$ elastic collisions along the SC.
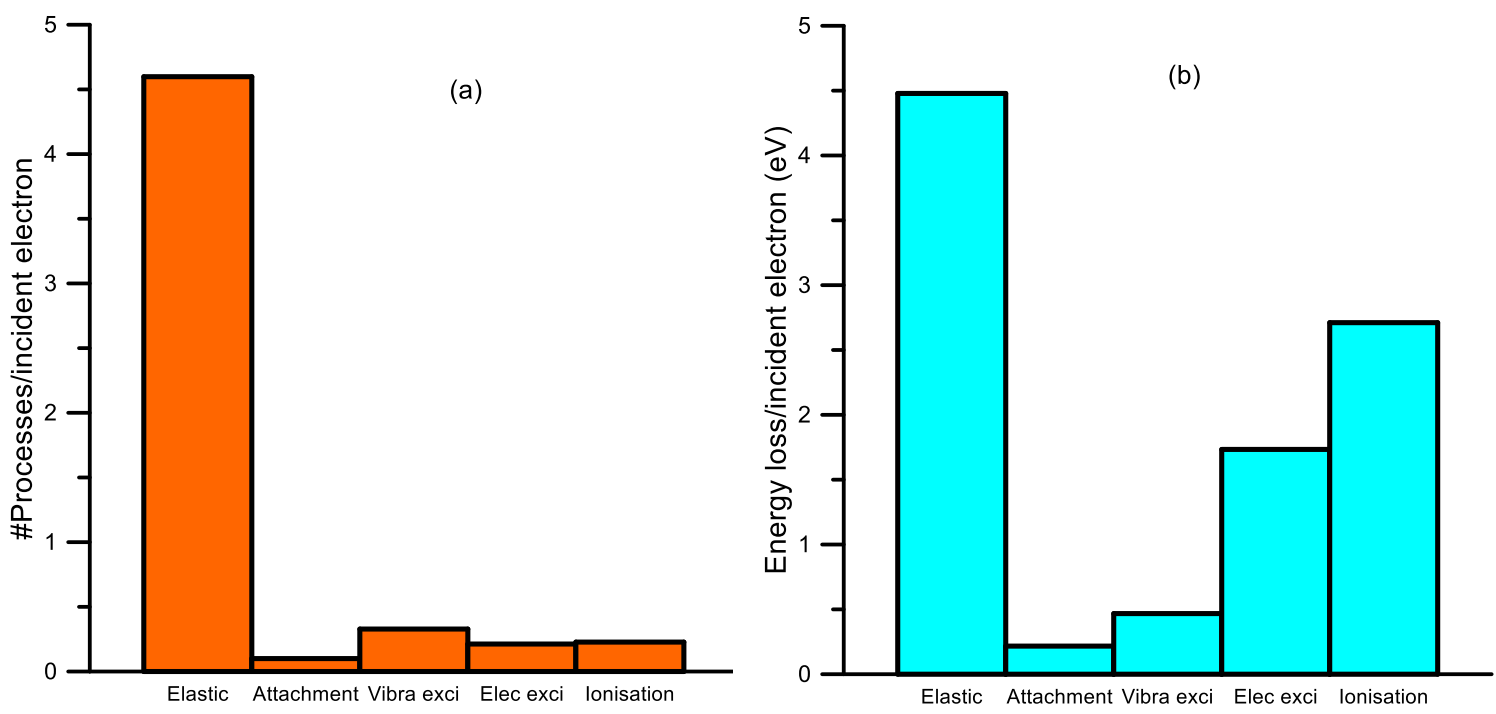

Fig.6. a) relative number of interaction processes per incident electron and b) energy loss in the axial direction per incident electron, for an initial kinetic energy of $15 \mathrm{eV}$.

As far as the energy loss is concerned, although the energy transferred to the medium via elastic processes is almost negligible (because of the projectile/target mass ratio), due to the intense axial magnetic field the kinetic energy loss along the parallel velocity component is still the main factor determining the energy distribution in the axial direction. This is clearly seen in Fig. 6 (b). Note that although electronic excitation and ionisation are not significant in terms of their number of interactions, due to their energy loss distribution functions (see Fig. 4) their contribution to the energy deposition is quite considerable. Considering that the simulation procedure had to cope with all the above noted difficulties, we can conclude that the very good agreement found between the measured and simulated electron energy distributions is valuable evidence for the reliability of the cross section data set presented in this experimental and theoretical study. Furthermore it is an important indication of the validity of the magnetically confined electron-beam technique used in the present measurements to assess the completeness of a cross section database.

\section{Concluding remarks}

We have presented the first measurements of the total electron scattering cross section from $\mathrm{pBQ}$ molecules in the energy range 1-200 eV. The utilised experimental technique was based on 
a state-of-the-art magnetically confined electron transmission-beam apparatus. After a comprehensive analysis of all possible random and systematic error sources, a total uncertainty limit within 4-7\% has been established for these experimental values. Measurements have been accompanied by a theoretical study of the interaction cross sections, by using two representative calculation methods in their respective energy range of applicability. For intermediate and high energies (15-200 eV), the IAM-SCAR+I procedure provided reliable (within $10 \%)$ differential and integral elastic scattering cross sections as well as integral electronic-state excitation and total ionisation cross sections. The self-consistency between the experimental and our theoretical data in this energy range allowed us to present a validated cross section set for energies above $15 \mathrm{eV}$, ready to be used for modelling applications. Note that this observation in $\mathrm{pBQ}$ is entirely consistent with what we have previously found for a range of other organic ring compounds. ${ }^{61-63}$ For lower energies (1-12 eV), elastic differential and integral scattering cross sections have been calculated by means of the SMCPP method at the 1ch-SEP (1-open channel and 88-closed channels) level of approximation. Here it is important to note that at this level of approximation the target is only slightly polarized due to the presence of the remaining 88-channels kept closed in the calculation for the elastic channel. Therefore, the assignment of the two structures appearing in the present SMCPP elastic ICS results should be confirmed by a new round of calculations to be performed with a more accurate treatment of the polarization effects. In fact, although resonances at 1.3 and $1.8 \mathrm{eV}$ in the measured TCS were identified as being due to electron attachment processes, there were some discrepancies in terms of the cross section values and resonance positions between our SMCPP 1ch-SEP calculation and the previous R-matrix result. ${ }^{20}$ When this observation is taken together with the fact of missing the $2.5 \mathrm{eV}$ resonance recently calculated ${ }^{21,22}$ and observed ${ }^{18}$ in photodetachment experiments, this suggests that further complementary techniques should be used to investigate this lower energy region.

Finally, an electron transport Monte Carlo simulation, for $15 \mathrm{eV}$ incident energy electrons, has been undertaken using the present cross section results as the required input probability distribution functions. The very good agreement found between the simulated and measured energy distributions of the transmitted electrons, provided strong evidence for the reliability of the present experimental and theoretical data in that low energy domain. Hence we believe we now have a self-consistent cross section data base for $\mathrm{pBQ}$, when simulating electron transport through it in the energy range $1-200 \mathrm{eV}$.

\section{Acknowledgements}

This study has partially been supported by the Spanish Ministry MINECO (Project FIS201680440). F.F.S. acknowledges the Portuguese National Funding Agency FCT through Researcher Contract No. IF-FCT IF/00380/2014, and, together with P.L.V., the Research Grant No. UID/FIS/00068/2013. M.J.B. thanks the Australian Research Council for funding through grants DP160102787 and DP180101655. Finally, M.A.P.L, M.T.N.V. (Grant I.D. 305672/2014-9), R.F.C. (Grant I.D. 313094/2017-9) and M.H.F.B. all acknowledge financial support from the Brazilian agency Conselho Nacional de Desenvolvimento Científico e Tecnológico (CNPq). These authors also wish to thank computational support from Centro de Computação John David Rogers (CCJDR) at Universidade Estadual de Campinas (UNICAMP). Finally, M.T.N.V. acknowledge Fundação de Amparo à Pesquisa do Estado de São Paulo (FAPESP) for funding.

\section{Conflicts of interest statement}

There are no conflicts of interest to declare 
1. M. Martínez-Cifuentes, R. Salazar, O. Ramírez-Rodríguez, B. Weiss-López and R. ArayaMaturana, Molecules, 2017, 22, 577.

2. D. B. Jones, R. F. da Costa, F. Kossoski, M. T. do N. Varella, M. H. F. Bettega, F. Ferreira da Silva, P. Limão-Vieira, G. Garcia, M. A. P. Lima, R. D. White and M. J. Brunger, J. Chem. Phys., 2018, 148, 124312.

3. D. B. Jones, R. F. da Costa, F. Kossoski, M. T. do N. Varella, M. H. F. Bettega, G. Garcia, F. Blanco, R. D. White, M. A. P. Lima and M. J. Brunger, J. Chem. Phys., 2018, 148, 204305.

4. B. Huskinson, M. P. Marshak, C. Suh, S. Er, M. R. Gerhardt, C. J. Galvin, X. Chen, A. Aspuru-Guzik, R. G. Gordon and M. J. Aziz, Nature, 2014, 505, 195.

5. Y. Ding and G. Yu, Angew. Chem. Int. Ed., 2016, 55, 4772.

6. B. Ómarsson and O. Ingólfsson, Phys. Cem. Chem. Phys., 2013, 15, 1658.

7. D. Dougherty and S. P. McGlynn, J. Am. Chem. Soc., 1977, 99, 3234.

8. J. F. Stanton, K. W. Sattelmeyer, J. Gauss, M. Allan, T. Skalicky and T. Bally, J. Chem. Phys., 2001, 115, 1.

9. C. R. Brundle, M. B. Robin and N. A. Kuebler, J. Am. Chem. Soc., 1972, 94, 1466.

10. L. Åsbrink, G. Bieri, C. Fridh, E. Lindholm and D. P. Chong, Chem. Phys., 1979, 43, 189.

11. N. Kishimoto, K. Okamura and K. Ohno, J. Chem. Phys., 2004, 120, 11062.

12. K. Piech, T. Bally, T. Ichino and J. Stanton, Phys. Chem. Chem. Phys., 2014, 16, 2011.

13. D. B. Jones, E. Ali, C. G. Ning, J. Colgan, O. Ingólfsson, D. H. Madison and M. J. Brunger, J. Chem. Phys., 2016, 145, 164306.

14. L. G. Christophorou, J. G. Carter and A. A. Christodoulides, Chem. Phys. Lett., 1969, 3, 237.

15. P. M. Collins, L. G. Christophorou, E. L. Chaney and J. G. Carter, Chem. Phys. Lett., 1970, 4, 646.

16. C. D. Cooper, W. T. Naff and R. N. Compton, J. Chem. Phys., 1975, 63, 2752.

17. A. Modelli and P. D. Burrow, J. Phys. Chem., 1984, 88, 3550.

18. C. W. West, J. N. Bull, E. Antonkov and J. R. Verlet, J. Phys. Chem. A, 2014, 118, 11346.

19. O. G. Khvostenko, P. V. Shchukin, G. M. Tuimedov, M. V. Muftakhov, E. E. Tseplin, S. N. Tseplina and V. A. Mazunov, Int. J. Mass Spectrom., 2008, 273, 69.

20. A. Loupas and J. D. Gorfinkiel, Phys. Chem. Chem. Phys., 2017, 19, 18252.

21. A. A. Kunitsa and K. B. Bravaya, J. Phys. Chem. Lett., 2015, 6, 1053.

22. A. A. Kunitsa and K. B. Bravaya, Phys. Chem. Chem. Phys., 2016, 18, 3454.

23. D. B. Jones, P. Limão-Vieira, M. Mendes, N. C. Jones, S. V. Hoffmann, R. F. da Costa, M. T. do N. Varella, M. H. F. Bettega, F. Blanco, G. García, O. Ingólfsson, M. A. P. Lima and M. J. Brunger, J. Chem. Phys., 2017, 146, 184303.

24. D. B. Jones, F. Blanco, G. García, R. F. da Costa, F. Kossoski, M. T. do N. Varella, M. H. F. Bettega, M. A. P. Lima, R. D. White, and M. J. Brunger, J. Chem. Phys., 2017, 147, 244304.

25. A. I. Lozano, K. Krupa, F. Ferreira da Silva, P. Limão-Vieira, F. Blanco, A. Muñoz, D. B. Jones, M. J. Brunger and G. García, Eur. Phys. J. D, 2017, 71, 226.

26. A. I. Lozano, J. C. Oller, K. Krupa, P. Limão-Vieira, F. Blanco, A. Muñoz, R. Colmenares and G. García, Rev. Sci. Instrum., 2018, 89, 063105.

27. M. C. Fuss, A. G. Sanz, F. Blanco, J. C. Oller, P. Limão-Vieira, M. J Brunger and G. García, Phys. Rev. A, 2013, 88, 042702.

28. A. G. Sanz, M. C. Fuss, F. Blanco, J. D. Gorfinkiel, A. Almeida, F. Ferreira da Silva, P. Limão-Vieira, M. J. Brunger and G. García, J. Chem. Phys., 2013, 139, 184310.

29. C. Szmytkowski and K. Maciag, Phys. Script., 1996, 54, 271.

30. Y. Itikawa, J. Phys. Chem. Ref. Data, 2006, 35, 31.

31. F. Blanco, L. Ellis-Gibbings and G. García, Chem. Phys. Lett., 2016, 645, 71. 
32. A. Traoré Dubuis, F. Costa, F. Ferreira da Silva, P. Limão-Vieira, J. C. Oller, F. Blanco and G. García, Chem. Phys. Lett., 2018, 699, 182.

33. N. Hishiyama, M. Hoshino, F. Blanco, G. García and H. Tanaka, J. Chem. Phys., 2017, 147, 224308.

34. F. Blanco, F. Ferreira da Silva, P. Limão-Vieira and G. García, Plasma Sources Sci. Technol., 2017, 26, 085004.

35. W. A. D. Pires, K. L. Nixon, S. Ghosh, R. F. C. Neves, H. V. Duque, R. A. R. Amorim, D. B. Jones, F. Blanco, G. García, M. J. Brunger and M. C. A. Lopes, Int. J. Mass Spectrom., 2017, 422, 32.

36. S. Ghosh, K. L. Nixon, W. A. D. Pires, R. A. R. Amorim, R. F. C. Neves, H. V. Duque, M. G. M. da Silva, D. B. Jones, F. Blanco, G. García, M. J. Brunger and M. C. A. Lopes, Int. J. Mass Spectrom., 2018, 430, 44.

37. K. Takasuka and V. McKoy, Phys. Rev. A, 1981, 24, 2473.

38. J. S. dos Santos, R. F. da Costa, and M. T. do N. Varella, J. Chem. Phys., 2012, 136, 084307.

39. M. H. F. Bettega, L. G. Ferreira, and M. A. P. Lima, Phys. Rev. A, 1993, 47, 1111.

40. R. F. da Costa, M. T. do N. Varella, M. H. F. Bettega and M. A. P. Lima, Eur. Phys. J. D, 2015, 69, 159.

41. M. J. Brunger, P. J. O. Teubner, A. M. Weigold and S. J. Buckman, J. Phys. B, 1989, 22, 1443.

42. H.-Y. Cheng and Y.-S. Huang, Phys. Chem. Chem. Phys., 2014, 16, 26306.

43. M. Allan, Chem. Phys., 1984, 84, 311.

44. J. Tennyson, Phys. Rep., 2010, 491, 29.

45. H. P. Trommsdorff, J. Chem. Phys., 1972, 56, 5358.

46. P. Brint, J.-P. Connerade, P. Tsekeris, A. Bolovinos and A. Baig, J. Chem. Soc. Faraday Trans., 1986, 2, 82, 367.

47. M. J. Brunger, Int. Rev. Phys. Chem., 2017, 36, 333.

48. D. A. Horke, Q. Li, L. Blancafort and R. R. Verlet, Nature Chem., 2013, 5, 711.

49. K. Scott, Chem. Eng. Process., 1992, 31, 21.

50. G. Kurisu, H. Zhang, J. L. Smith, W. Cramer, Science, 2003, 302, 1009.

51. T. M. Iverson, C. Luna-Chavez, G. Cecchini, D. C. Rees, Science, 1999, 284, 1961.

52. B. Boudaïfa, P. Cloutier, D. Hunting, M. H. Huels and L. Sanche, Science, 2000, 287, 1658.

53. J. C. Butcher and H. Messel, Phys. Rev., 1958, 112, 2096.

54. G. García Gómez-Tejedor, and M. C. Fuss (eds.), Radiation Damage in Biomolecular Systems, (Springer: London, 2012).

55. F. Blanco, A. Muñoz, D. Almeida, F. Ferreira da Silva, P. Limão-Vieira, M. C. Fuss, A. G. Sanz and G. García, Eur. Phys. J., 2013, D 67, 199.

56. M. C. Fuss, L. Ellis-Gibbings, D. B. Jones, M. J. Brunger, F. Blanco, A. Muñoz, P. LimãoVieira and G. García, J. Appl. Phys., 2015, 117, 214701.

57. M. J. Brunger, K. Ratnavelu, S. J. Buckman, D. B. Jones, A. Muñoz, F. Blanco and G. García, Eur. Phys. J. D, 2016, 70, 46.

58. T. P. R. Kumar, B. Brynjarsson, F. H. Ómarsson, M. Hoshino, H. Tanaka, P. Limão-Vieira, D. B. Jones, M. J. Brunger and O. Ingólfsson, Int. J. Mass Spectrom., 2018, 426, 12.

59. F. H. Ómarsson, B. Reynisson, M. J. Brunger, M. Hoshino, H. Tanaka, P. Limão-Vieira and O. Ingólfsson, Int. J. Mass Spectrom., 2014, 365, 275.

60. S. Engmann, M. Stano, P. Papp, M. J. Brunger, Š. Matejčík and O. Ingólfsson, J. Chem. Phys., 2013, 138, 044305.

61. D. B. Jones, R. F. Da Costa, M. T. do N. Varella, M. H. F. Bettega, M. A. P. Lima, F. Blanco, G. García and M. J. Brunger, J. Chem. Phys., 2016, 144, 144303. 
62. R. F. da Costa, E. M. de Oliveira, M. H. F. Bettega, M. T. do N. Varella, D. B. Jones, M. J. Brunger, F. Blanco, R. Colmenares, P. Limão-Vieira, G. García and M. A. P. Lima, J. Chem. Phys., 2015, 142, 104304.

63. H. V. Duque, L. Chiari, D. B. Jones, P. A. Thorn, Z. Pettifer, G. B. da Silva, P. Limão-Vieira, D. Duflot, M.- J. Hubin-Franskin, J. Delwiche, F. Blanco, G. García, M. C. A. Lopes, K. Ratnavelu, R. D. White and M. J. Brunger, Chem. Phys. Lett., 2014, 608, 161. 